\title{
MANEJO CONSERVADOR EN UNA PACIENTE CON EMBARAZO ECTÓPICO CERVICAL EN NARIÑO, COLOMBIA: REPORTE DE CASO Y REVISIÓN DE LA LITERATURA
}

\section{Conservative management in a patient with cervical ectopic pregnancy in Nariño, Colombia: Case report and review of the literature}

Henry Hernán Bolaños-Bravo, MD, MSc ${ }^{1}$; Andrés Ricaurte-Fajardo, $\mathrm{MD}^{2}$; Fabio Zarama-Márquez, MD, MSc ${ }^{1}$; Andrés Ricaurte-Sossa, $M D, M S c^{1}$; Ruth Fajardo-Rivera, $M D^{3}$; Rubén Chicaiza-Maya, $M D^{3}$; Carlos Andrés Guerrero-Mejía, $M^{4}$

Recibido: 18 de abril de 2019 / Aceptado: 21 de diciembre de 2019

\section{RESUMEN}

Objetivos: reportar el caso de un embarazo cervical (EC) que recibió manejo conservador exitoso y realizar una revisión de la literatura sobre el tratamiento médico y quirúrgico conservador.

Materiales y métodos: se presenta el caso de una paciente con embarazo cervical, quien recibió manejo farmacológico con metotrexate (MTX) y posterior legrado con evolución clínica satisfactoria. Se realizó una búsqueda de artículos en Medline vía PubMed, LILACS, SciElo y Google académico con los términos: "cervical ectopic pregnancy", "conservative treatment", "curettage", "methotrexate",

* Correspondencia: Andrés Ricaurte-Fajardo, Carrera 7 No. 40-62, Bogotá (Colombia). Teléfono: (031)3208320.

andres.ricaurte@javeriana.edu.co

Especialista en Ginecología y Obstetricia, Departamento de Ginecología y Obstetricia, Hospital Universitario Departamental de Nariño, San Juan de Pasto (Colombia).

Médico Interno, Hospital Universitario San Ignacio, Pontificia Universidad Javeriana, Bogotá D.C (Colombia).

Médico interno, Hospital Universitario Departamental de Nariño, Universidad Cooperativa de Colombia, San Juan de Pasto (Colombia).

4 Médico interno, Hospital Universitario Departamental de Nariño, Fundación Universitaria San Martín, San Juan de Pasto (Colombia). "uterine artery embolization" "hysteroscopy". Se seleccionaron reportes y series de caso, pacientes con embarazo cervical diagnosticado por ultrasonido, de cualquier edad gestacional, sometidas tratamiento médico o quirúrgico conservador.

Resultados: se incluyeron 22 estudios; se identificaron 95 pacientes con EC tratados con MTX, con tratamiento exitoso en 93. La complicación más frecuente fue la hemorragia en $12 \%$; el 26\% requirió tratamiento quirúrgico complementario. Cada vez más, la embolización de arterias uterinas (EAU) se realiza de manera preventiva (7 casos) antes del legrado o del tratamiento con MTX. La histeroscopia es otra alternativa reciente (20 casos). En 2 casos se requirió histerectomía abdominal, uno de los cuales fue un embarazo ístmico cervical. Conclusiones: el tratamiento con MTX sigue siendo el más frecuentemente utilizado. La dilatación y el curetaje con taponamiento endocervical puede ser una opción por considerar en el manejo de urgencia del EC en instituciones de atención primaria. En instituciones donde se dispone de tecnologías de 
alta complejidad, la embolización de arterias uterinas previa a los procedimientos quirúrgicos y la histeroscopia son opciones que se deben considerar. Dado que actualmente es posible el diagnóstico temprano del EC, se requieren estudios multicéntricos que comparen las diferentes alternativas de manejo para una mejor evaluación de su seguridad y efectividad.

Palabras clave: embarazo ectópico; tratamiento conservador; legrado; gonadotropina coriónica; fertilidad (DeCS).

\section{ABSTRACT}

Objectives: To report a case of cervical pregnancy (CP) treated successfully with a conservative approach, and to conduct a review of the literature regarding conservative medical and surgical treatment.

Materials and Methods: Patient with cervical pregnancy treated pharmacologically with methotrexate (MTX) followed by dilation and curettage, with a satisfactory clinical course. A search of articles was conducted in Medline via PubMed, LILACS, SciElo and Google Scholar using the terms "cervical ectopic pregnancy," "conservative treatment," “curettage,» "methotrexate," "uterine artery embolization," "hysteroscopy." Reports and case series were selected of patients with cervical pregnancy diagnosed on ultrasound at any gestational age, subjected to conservative medical or surgical treatment.

Results: A total of 22 studies were included; 95 patients with CP treated with MTX were identified, 93 of them successfully treated. The most frequent complication was bleeding in $12 \%$; $26 \%$ required complementary surgical treatment. Increasingly, uterine artery embolization (UAE) is carried out preventatively (7 cases) before curettage or treatment with MTX. The hysteroscopy is another recent alternative (20 cases). Abdominal hysterectomy was required in two cases, one of which was a cervico-isthmic pregnancy.
Conclusions: Treatment with MTX continues to be the most frequent strategy. Dilation and curettage with endocervical plugging may be an option to consider in the emergency management of EP in primary care institutions. In institutions equipped with high complexity technology, uterine artery embolization before the surgical procedure and histeroscopy are options to be considered. Considering that early diagnosis of EP is now possible, multi-center studies comparing different management options are needed for better assessment of their safety and effectiveness.

Key words: Ectopic pregnancy; conservative treatment; curettage; chorionic gonadotropin; fertility (MeSH).

\section{INTRODUCCIÓN}

El embarazo ectópico cervical se define como aquel que se implanta en el cuello uterino por debajo del orificio cervical interno (1). Schneider et al. (2) atribuyen a Everard Home, en 1817, la primera mención del embarazo ectópico cervical, además indican que el término fue acuñado por Rokitansky en 1860. Schneider refiere que en 1911, Rubin estableció cuatro criterios para el diagnóstico de esta condición, a saber: la existencia de glándulas cervicales opuestas a la unión placentaria, presencia de una íntima unión entre la placenta y el cérvix, la localización de la unión placentaria al cérvix por debajo de la entrada de los vasos uterinos o por debajo de la reflexión peritoneal anterior y posterior de la superficie del útero, y la no existencia de elementos fetales en el área del cuerpo del útero. La frecuencia de este tipo de ectópicos varía entre 1:1500 a 1:18.000 (3-6). Por otra parte, Samal cita a Celik quien informa una frecuencia de 1:95.000 (7) de todos los embarazos, que corresponde al 0,1\% de todos los embarazos ectópicos, esta es la presentación menos frecuente (8).

En cuanto a los factores de riesgo, dado lo poco frecuente del evento, no hay estudios que permitan establecer asociaciones con la precisión requerida, 
sin embargo, hay factores que se han asociado como: la fertilización in vitro, la enfermedad pélvica inflamatoria y el curetaje endometrial, aunque la evidencia es controvertida (9).

El diagnóstico del embarazo ectópico cervical es más exacto hoy en día gracias a la disponibilidad de mediciones cuantitativas de gonadotropina coriónica humana en suero (hCG), imágenes del ultrasonido transvaginal y de la resonancia magnética (10). En la actualidad, los criterios ecográficos que más se utilizan son los descritos por Ushakov et al. (4), quien propone como criterios mayores: a) localización intracervical de un saco de gestación o masa trofoblástica, b) orificio cervical interno cerrado y c) invasión local del tejido endocervical por el trofoblasto; como signos adicionales: d) visualización de estructuras embrionarias o fetales en el saco gestacional ectópico y presencia de fetocardia, e) cavidad uterina vacía, f) decidualización endometrial, g) útero en reloj de arena y h) presencia de flujo arterial peritrofoblástico intracervical con el Doppler a color. Sin embargo, hay falsos positivos por abortos espontáneos detectados a su paso a través del cérvix (9).

Históricamente, los embarazos ectópicos cervicales eran diagnosticados de forma intraoperatoria durante la realización de un legrado, por la presencia de una importante hemorragia que amenazaba la vida de la paciente y donde la alternativa que le quedaba al ginecobstetra era la histerectomía (11). Sin embargo, a partir de los años ochenta (12) y con el advenimiento de los medios diagnósticos mencionados, que han permitido la detección temprana de esta condición, ha habido un cambio significativo de dicho enfoque y hoy en día se utilizan modalidades de tratamiento más conservadoras con base en el tratamiento médico con metotrexate (MTX) (sistémico o local), taponamiento intracervical con balones de sonda de Foley, legrado e inyección local de prostaglandinas, cerclaje cervical e inyecciones intracervicales de agentes vasoconstrictores, ligadura de arteria uterina asistida por laparoscopia combinada con resección endocervical histeroscópica, resección histeroscópica combinada con embolización de arterias uterinas (EAU) que buscan conservar el útero y la fertilidad en la mujer (4, 9, 13). Ushakov et al., describieron las condiciones para iniciar manejo conservador, dentro de las cuales se incluyen: tener un diagnóstico fiable de embarazo ectópico cervical; que la paciente se encuentre hemodinámicamente estable, sin sangrado o con sangrado leve; edad gestacional menor a 10 semanas por fecha de última menstruación; recuento plaquetario normal; funciones hepática y renal normales (4). La escogencia entre dosis única o esquema multidosis de MTX depende de las condiciones de la paciente. Murji, citando a Hung, describe los criterios para el uso de múltiples dosis de MTX: un nivel de b-HCG > $10.000 \mathrm{mUI} / \mathrm{ml}$, edad gestacional mayor de 9 semanas, presencia de actividad cardiaca embrionaria o fetal y una longitud cefalocaudal mayor de $10 \mathrm{~mm}$ en la ecografía transvaginal (14). En presencia de actividad cardiaca embrionaria o fetal se recomienda el uso de inyecciones intrasaculares de cloruro de potasio (KCL) o MTX (15-17).

El tratamiento médico exitoso se define como la preservación del útero con MTX, incluso si se requirió de un procedimiento menor como la dilatación y curetaje, la angio-embolización o la histeroscopia, y se considera como tratamiento médico fallido cuando la paciente debe ser llevada a histerectomía o cuando, aunque exista preservación uterina, se haya requerido de un procedimiento mayor como laparotomía, ligadura de arterias uterinas o histerotomía (15).

Tenemos pues que en el embarazo ectópico cervical el tratamiento conservador es hoy en día el estándar de manejo, sin embargo, es una condición seria que amenaza la vida de la paciente, sobre todo en casos en los que no se ha realizado un diagnóstico temprano, por tanto, el médico general y el obstetra que atiende urgencias deben tener en mente esta posibilidad diagnóstica, sobre todo en 
casos de hemorragia genital masiva, como también el médico que hace atención primaria y control prenatal, para realizar un diagnóstico temprano de la entidad que permita un manejo conservador. Es poca la información sobre el manejo actual de la entidad y sus complicaciones, desde la revisión de la literatura realizada por Ushakov en 1997 (4), que es un referente en la entidad. Por esta razón, se presenta el caso de una paciente con embarazo ectópico cervical con el objetivo de hacer una revisión de la literatura publicada desde el año 2000 en cuanto al tratamiento y sus complicaciones.

\section{PRESENTACIÓN DEL CASO}

Mujer de 30 años, que consultó por cuadro de tres horas de evolución consistente en sangrado genital moderado indoloro al Hospital Universitario Departamental de Nariño, institución de referencia, perteneciente al régimen subsidiado por el Estado en el Sistema General de Seguridad Social en Colombia, ubicada en la ciudad de Pasto, en la región suroccidental de Colombia. Tenía amenorrea de 5 semanas y traía un reporte extrainstitucional de prueba de embarazo positiva. La paciente tenía un antecedente de cesárea de hacía 7 años, e historia familiar de diabetes mellitus. Al examen físico se encontró presión arterial: 118/74 mm/Hg, frecuencia cardiaca: 90 latidos por minuto, frecuencia respiratoria: 18 respiraciones por minuto, saturación arterial de oxígeno: $96 \%$ con fracción inspirada de oxígeno de $21 \%$, sin signos de irritación peritoneal y con presencia de sangrado al examen ginecológico. Se tomó un hemograma en el cual se reportó hematocrito: 41,4\%, hemoglobina: 14,1 g/dl, leucocitos: 9800 , neutrófilos: $60 \%$ y subunidad Beta de la gonadotrofina coriónica humana $(\beta \mathrm{GCH})$, que mostró un título de $4158 \mathrm{mg} / \mathrm{dl}$; además, se realizó un ultrasonido transvaginal que mostró hallazgos sugestivos de embarazo temprano sin evidencia de saco gestacional, por lo cual se consideró como impresión diagnóstica una amenaza de aborto, teniendo como diagnóstico diferencial embarazo ec- tópico. Se dio egreso y se solicitó una nueva $\beta \mathrm{GCH}$ con recomendaciones de signos de alarma. Cuatro días después, la paciente regresó a control con sangrado genital, trae nuevo resultado de $\beta \mathrm{GCH}$ : $16.189 \mathrm{mUI} / \mathrm{ml}$, y ultrasonido transvaginal en el que se describe un saco gestacional único localizado a nivel del endocérvix uterino con adecuada reacción decidual, por debajo de la inserción de las arterias uterinas y del orificio cervical interno, de aspecto cerrado (figura 1), sin interrupción de la continuidad de la línea endometrial-cervical, y ausencia del "signo de deslizamiento". A los dos días se hizo nuevo ultrasonido transvaginal que mostró persistencia de la imagen del saco a nivel cervical, con un embrión único, con embriocardia positiva, que cumplía con los criterios ecográficos de Ushakov (4), lo cual confirmó el embarazo ectópico cervical.

La paciente manifestó deseo de fertilidad en el futuro, por lo cual se decidió iniciar manejo conservador, explicando la posibilidad de un eventual manejo quirúrgico, en caso de ser necesario, por inestabilidad hemodinámica. Se inició manejo con metotrexato a dosis de 60 mg vía intravenosa (IV)/ día, dosis única, y posteriormente se repitió la dosis a los 3, 5 y 7 días, asociado a $15 \mathrm{mg}$ de folinato de calcio vía oral en los días 4, 6 y 8 de tratamiento. Al quinto día de tratamiento, los valores de la $\beta \mathrm{GCH}$ de control se encontraron duplicados (35.199 mUI/ $\mathrm{ml})$. Se realizó una resonancia magnética pélvica en la que se documentó una imagen de aspecto quístico, con reacción decidual compatible con un embarazo ectópico cervical e invasión trofoblástica del estroma cervical anterior, respetando la pared vesical (figura 2). En junta médico-quirúrgica se decidió realizar legrado obstétrico tres horas después de la administración de misoprostol y ácido tranexámico.

En sala de cirugía se realizó examen ginecológico, a la especuloscopia se observó saco gestacional a través del orificio cervical externo, se pinzó el labio anterior del cuello con un tenáculo y con la ayuda de una pinza de falsos gérmenes se realizó extracción 


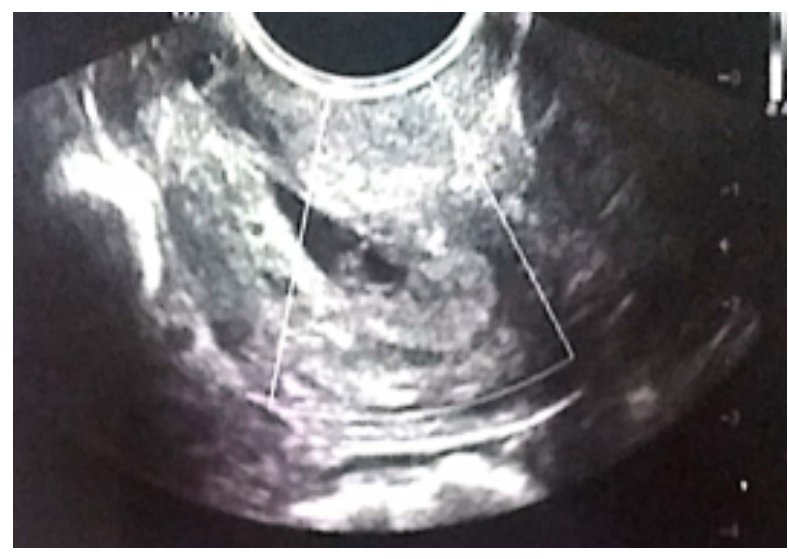

Figura 1. Ecografía pélvica transvaginal A nivel endocervical (distal al orificio cervical interno) se observa saco gestacional único irregular con pérdida de la turgencia y elongación distal que mide $18 \times 7 \mathrm{~mm}$, en cuyo interior se identifica saco vitelino de $3 \mathrm{~mm}$. La imagen es compatible con un embarazo ectópico cervical de 5 semanas, 6 días

quirúrgica del saco gestacional; a continuación, se procedió a realizar legrado del cuello uterino y del cuerpo del útero con cureta cortante, no se presentó sangrado importante a través del cuello uterino. Finalmente, en el canal endocervical se colocó una sonda de Foley inflando su balón con 15 cc de solución salina para presionar y prevenir sangrado proveniente del lecho intervenido, posterior al procedimiento. Después del mismo, por alto riesgo de deterioro hemodinámico, la paciente es trasladada de manera preventiva a la unidad de cuidados intensivos para vigilancia estricta del patrón de sangrado uterino; el espécimen quirúrgico fue enviado a patología.

En el posoperatorio, a las 24 horas, se disminuyó progresivamente 5 cc cada 12 horas la presión del balón de la sonda de Foley, sin que se presentara sangrado uterino, con valores de hematocrito y hemoglobina de comportamiento estable. A los dos días del procedimiento los niveles de la bHCG disminuyeron a $5282 \mathrm{mUI} / \mathrm{ml}$, al cuarto día disminuyeron a $1823 \mathrm{mUI} / \mathrm{ml}$. La paciente fue dada de alta 5 días después del legrado y al sexto día tuvo

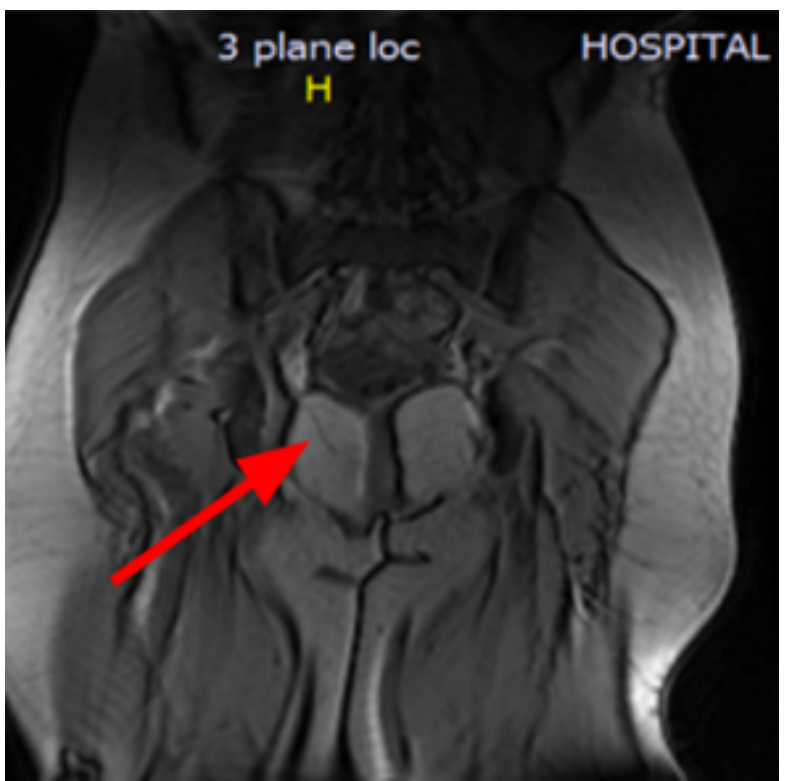

Figura 2. Resonancia magnética pélvica

Imagen redondeada de señal alta, localizada en el cérvix, con diámetros mayores de $22 \times 20 \mathrm{~mm}$, con halo hipointenso redondeado, que muestra realce periférico en anillo en la fase contrastada, compatible con embarazo ectópico cervical

un valor de bHCG de $809 \mathrm{mUI} / \mathrm{ml}$. Otro control de bHCG realizado 17 días posprocedimiento reportó un resultado de $16 \mathrm{mUI} / \mathrm{ml}$. El resultado de histopatología informó un saco gestacional colapsado, con tejido de embrión sin inflamación, con vellosidades coriales inmaduras. Tres meses después, se realiza histeroscopia en la que se evidenció cavidad uterina y ostium normales, canal cervical normal y orificio cervical interno sin lesiones, con lo que se confirma el éxito del manejo conservador médicoquirúrgico con preservación uterina y evolución clínica satisfactoria.

Aspectos éticos. La paciente firmó el consentimiento informado autorizando a los autores la publicación del caso.

\section{MATERIALES Y MÉTODOS}

Se realizó una búsqueda de artículos en Medline vía PubMed, en la librería en línea SciElo, LILACS y en el buscador Google académico con los térmi- 
nos: "cervical ectopic pregnancy", "conservative treatment", "curettage", "methotrexate", "uterine artery embolization", "hysteroscopy", con sus correspondientes términos en español, así como una búsqueda en bola de nieve a partir de los estudios identificados. Se seleccionaron reportes y series de casos que hubieran incluido pacientes con embarazo ectópico cervical diagnosticados por ultrasonido, de cualquier edad gestacional, y que hubieran recibido tratamiento médico con MTX, manejo conservador quirúrgico con dilatación y curetaje, histeroscópico o con EAU, publicados en inglés y español en el periodo 2000 a 2019 y en los que se dispuso del texto completo. Se evaluaron los siguientes aspectos de los estudios: diseño, tipo de tratamiento médico, quirúrgico, país donde se hizo el manejo del caso, semanas de gestación al diagnóstico; en el tratamiento médico: medicamentos utilizados, dosis y vía de administración; en el tratamiento quirúrgico: técnica quirúrgica empleada, complicaciones, resultado materno (éxito o fracaso), tiempo de seguimiento y necesidad de tratamiento médico-quirúrgico.

\section{RESULTADOS}

A partir de la búsqueda en la literatura se encontraron 130 títulos, de estos se identificaron 22 estudios que correspondieron a series y reportes de caso que cumplieron con los criterios de inclusión: 3 fueron descritos como estudios prospectivos (18-20), 6 estudios retrospectivos $(5,14,21-24), 3$ series de casos (25-27), y 10 reportes de caso (7, 16, 28-35). Un total de 15 estudios evaluaron el tratamiento médico $(5,7,14,18,21-23,25,28-33,35)$ y 9 el tratamiento quirúrgico $(7,16,19,20,24,26,27,34,35)$.

Los textos seleccionados fueron publicados por grupos de varios países como Taiwán (20), India (7, 35), Grecia (18), Canadá (5, 14, 31), Turquía (22), Italia (16, 19), Chipre (26), Egipto (26), Estados Unidos (23, 25, 34), China (24), Corea del Sur (27), España (33), México (29,), Venezuela (30), Cuba (32) y Colombia (28).

\section{Tratamiento médico}

Medicamentos utilizados. El metotrexate (MTX) fue utilizado como pilar del manejo en el abordaje inicial en los siguientes estudios (tabla 1).

Vela et al. (5) informan un estudio retrospectivo de doce pacientes con EC, cinco de los cuales recibieron tratamiento con MTX, con una edad gestacional que varió entre las 6,5 y las 9,1 semanas. Cuatro recibieron como manejo inicial MTX en dosis única y otra recibió MTX local e intramuscular. Una paciente respondió adecuadamente a la dosis única de MTX, mientras que las otras 4 requirieron tratamiento adicional con una segunda dosis de MTX (1 paciente), embolización de la arteria uterina (2 pacientes), curetaje y ligadura vascular (1 paciente). La paciente restante cursó simultáneamente con un embarazo intrauterino y recibió tratamiento con MTX y EAU con expulsión de ambos sacos gestacionales sin requerir otras intervenciones. En todos los casos, se hizo seguimiento aproximadamente a un año y el manejo conservador fue exitoso en todos casos.

Kirk et al. (21) exponen un estudio retrospectivo de siete pacientes con EC con edad gestacional de 7 a 12 semanas, de las cuales 6 recibieron MTX, dado que una paciente recibió manejo expectante. En este estudio se determinó el uso de MTX en monodosis en aquellos embarazos cervicales sin actividad cardiaca fetal, mientras que en los que estaba presente la actividad cardiaca fetal se indicó el uso del protocolo de monodosis o de multidosis con rescates de ácido folínico o MTX intraamniótico. Tres casos recibieron una sola dosis, una multidosis y otra multidosis oral e intraamniótica con resultados exitosos. En un caso se requirió la aplicación de CLK intraamniótico complementario. El seguimiento se hizo en un promedio de 63 días.

Uludag et al. (22) revisaron diez pacientes entre 23 y 40 años con EC, con edad gestacional entre 6,2 y 8,2 semanas, de las cuales seis pacientes recibieron MTX sistémico multidosis y 4 pacientes inyección local intraamniótica de MTX. El manejo se consi- 


\begin{tabular}{|c|c|c|c|c|c|c|c|c|c|}
\hline \multicolumn{10}{|c|}{ Revisión de la literatura, 2000-2019 } \\
\hline$\stackrel{0}{\stackrel{2}{2}}$ & 送 & 气 & 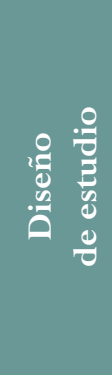 & 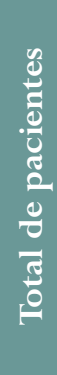 & 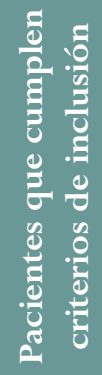 & 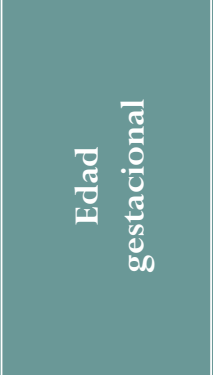 & 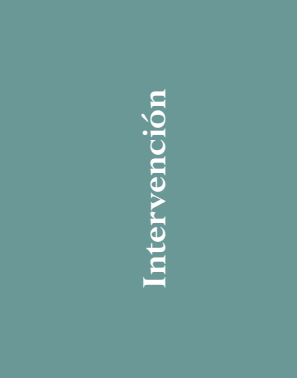 & 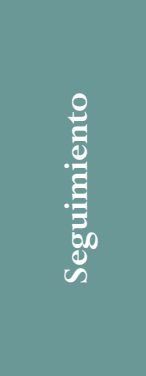 & 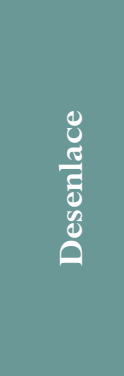 \\
\hline $\begin{array}{l}\text { Vela G, } \\
\text { et al. (5) }\end{array}$ & 2007 & Canadá & $\begin{array}{l}\text { Retros- } \\
\text { pectivo }\end{array}$ & 12 & 5 & $\begin{array}{l}\text { 1.6.5 semanas } \\
\text { 2.6.6 semanas } \\
\text { 3.8.8 semanas } \\
\text { 4.8.8 semanas } \\
\text { 5.9.1 semanas }\end{array}$ & $\begin{array}{l}4 \text { pacientes: } \\
\text { Monodosis de } \\
\text { MTX } 50 \mathrm{mg} / \mathrm{m} 2 \mathrm{IM} \\
1 \text { paciente: MTX local } \\
\text { e IM }\end{array}$ & 1 año & Exitoso \\
\hline $\begin{array}{l}\text { Kirk, } \\
\text { et al. }(21)\end{array}$ & 2006 & Inglaterra & $\begin{array}{l}\text { Retros- } \\
\text { pectivo }\end{array}$ & 7 & 6 & $\begin{array}{l}1.6 \text { semanas } \\
2.6 \text { semanas } \\
3.6 \text { semanas } \\
4.7 \text { semanas } \\
5.11 \text { semanas } \\
6.11 \text { semanas }\end{array}$ & $\begin{array}{l}3 \text { pacientes: mono- } \\
\text { dosis de MTX } 50 \mathrm{mg} / \\
\text { m2 IM, } 1 \text { paciente: } \\
\text { multidosis MTX } 1 \\
\text { mg/kg IM en los días } \\
\text { 1,3,5 más rescate de } \\
\text { ácido folínico } 0,1 \mathrm{mg} / \\
\text { kg los días } 2,4,6, \\
1 \text { paciente: MTX } \\
\text { intraamniótico, } 1 \text { pa- } \\
\text { ciente: multidosis de } \\
\text { MTX IM más cloruro } \\
\text { de potasio intraam- } \\
\text { niótico } 5 \text { mmol/L }\end{array}$ & $\begin{array}{l}\text { Promedio } \\
\text { de } 63 \text { días } \\
\text { (rango } \\
34-139)\end{array}$ & Exitoso \\
\hline $\begin{array}{l}\text { Uludag, } \\
\text { et al. ( } 22)\end{array}$ & 2017 & Turquía & $\begin{array}{l}\text { Retros- } \\
\text { pectivo }\end{array}$ & 10 & 10 & $\begin{array}{l}\text { 1.6.2 semanas } \\
\text { 2.6.4 semanas } \\
\text { 3.6.5 semanas } \\
\text { 4.6.6 semanas } \\
\text { 5.6.2 semanas } \\
\text { 6.6.5 semanas } \\
\text { 7.7.0 semanas } \\
\text { 8. } 6.3 \text { semanas } \\
\text { 9. } 7.5 \text { semanas } \\
\text { 10. 8.2 semanas }\end{array}$ & $\begin{array}{l}6 \text { pacientes: MTX } \\
\text { sistémico en mul- } \\
\text { tidosis de } 1 \mathrm{mg} / \mathrm{kg} \\
\text { alternados con } 0,1 \\
\mathrm{mg} / \mathrm{kg} \text { de leucovo- } \\
\text { rina IM ( } 1 \text { requirió } \\
\text { cambio a tratmiento } \\
\text { local de MTX), } 4 \text { pa- } \\
\text { cientes: inyección de } \\
\text { MTX local intraam- } \\
\text { niótico } 50 \mathrm{mg}\end{array}$ & $\begin{array}{c}12 \\
\text { semanas } \\
\text { en } \\
\text { promedio }\end{array}$ & Exitoso \\
\hline $\begin{array}{l}\text { Martínez } \\
\text { et al. ( } 28)\end{array}$ & 2017 & Colombia & $\begin{array}{l}\text { Reporte } \\
\text { de caso }\end{array}$ & 1 & 1 & Desconocida & $\begin{array}{l}\text { MTX sistémico multi- } \\
\text { dosis de } 1 \mathrm{mg} / \mathrm{kg} \text { más } \\
\text { ácido folínico }\end{array}$ & 2 meses & Exitoso \\
\hline $\begin{array}{l}\text { De la } \\
\text { Loza } \\
\text { Cava, } \\
\text { et al. (29) }\end{array}$ & 2012 & México & $\begin{array}{l}\text { Reporte } \\
\text { de caso }\end{array}$ & 1 & 1 & 14 semanas & $\begin{array}{l}\text { MTX monodosis de } \\
50 \mathrm{mg} \mathrm{IM}\end{array}$ & 3 días & $\begin{array}{l}\text { Histerec- } \\
\text { tomía }\end{array}$ \\
\hline $\begin{array}{l}\text { Figueroa } \\
\text { Solano, } \\
\text { et al. }(30)\end{array}$ & 2012 & Venezuela & $\begin{array}{l}\text { Reporte } \\
\text { de caso }\end{array}$ & 1 & 1 & Desconocida & $\begin{array}{l}\text { MTX en multidosis } \\
\text { IV }\end{array}$ & 39 días & $\begin{array}{l}\text { Histerec- } \\
\text { tomía }\end{array}$ \\
\hline $\begin{array}{l}\text { Weibel, } \\
\text { et al. (31) }\end{array}$ & 2012 & Canadá & $\begin{array}{l}\text { Reporte } \\
\text { de caso }\end{array}$ & 2 & 2 & $\begin{array}{l}\text { 1.5.6 semanas } \\
2.7 .4 \text { semanas }\end{array}$ & $\begin{array}{l}\text { MTX en multidosis } \\
\text { de } 50 \mathrm{mg} / \mathrm{m} 2 \mathrm{IM} 4 \\
\text { días y ácido folínico } \\
\text { vía oral } 5 \mathrm{mg} \text { diarios }\end{array}$ & 2 meses & Exitoso \\
\hline
\end{tabular}




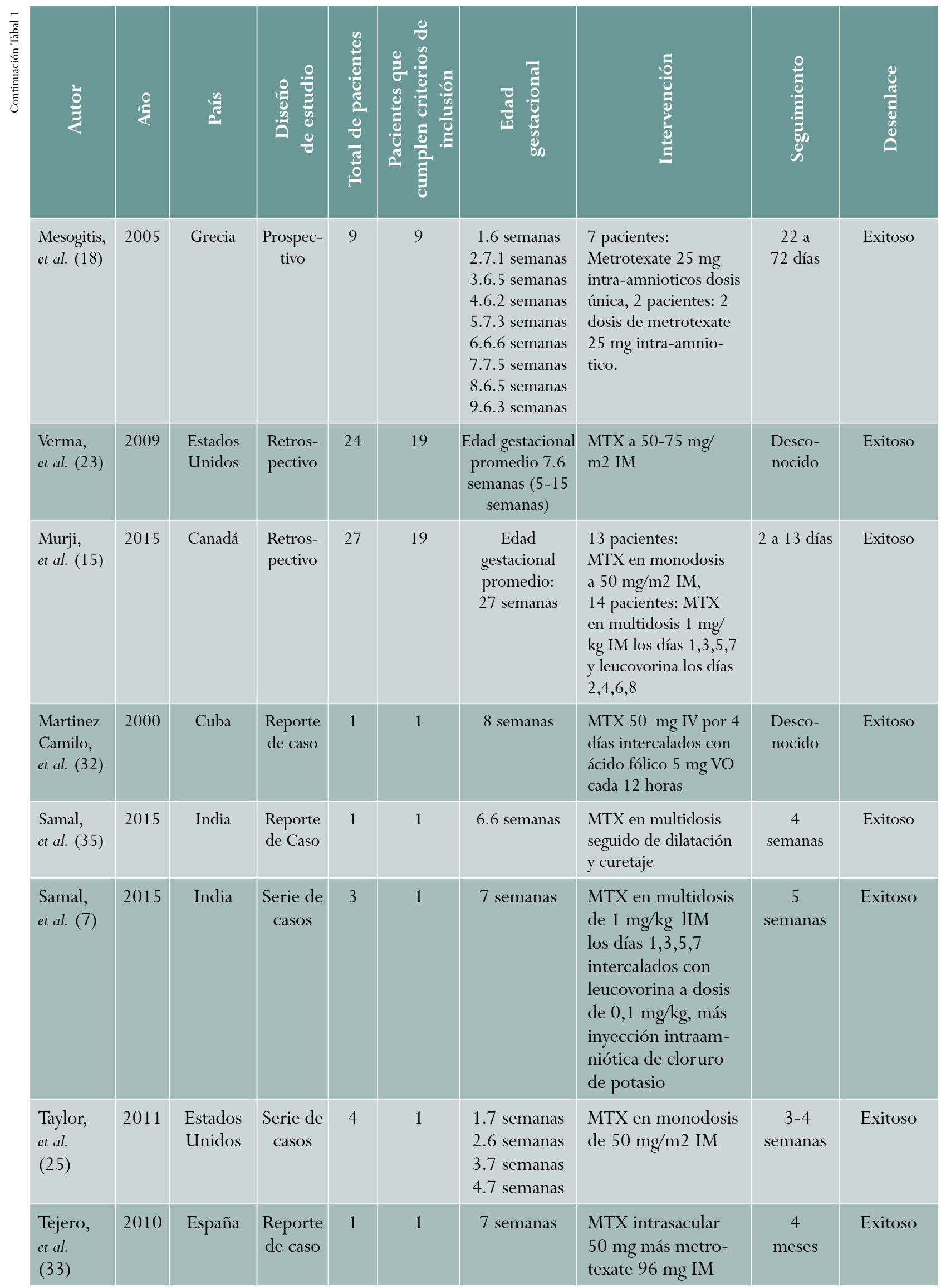




\begin{tabular}{|c|c|c|c|c|c|c|c|c|c|}
\hline \multicolumn{10}{|c|}{ Tratamiento quirúrgico conservador } \\
\hline \multicolumn{10}{|c|}{ Dilatación y curetaje } \\
\hline$\frac{\mathfrak{3}}{2}$ & 量 & $\stackrel{\mathscr{E}}{\mathrm{E}}$ & 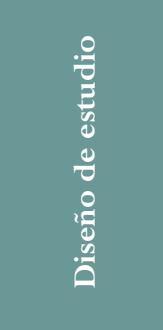 & 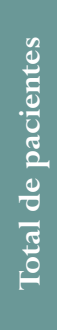 & 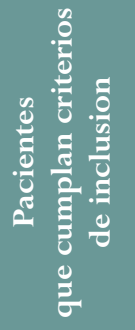 & 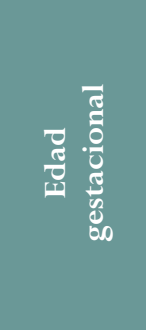 & 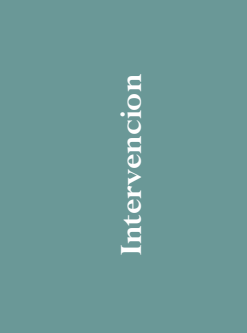 & 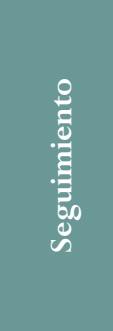 & 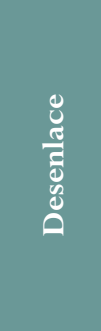 \\
\hline $\begin{array}{l}\text { De la Vega, } \\
\text { et al. ( } 34 \text { ) }\end{array}$ & 2007 & $\begin{array}{l}\text { Estados } \\
\text { Unidos }\end{array}$ & $\begin{array}{l}\text { Reporte } \\
\text { de Caso }\end{array}$ & 1 & 1 & $\begin{array}{c}7 \\
\text { semanas }\end{array}$ & $\begin{array}{l}\text { Dilatación y } \\
\text { curetaje más car- } \\
\text { boprost y tapona- } \\
\text { miento con balón }\end{array}$ & 7 días & Exitoso \\
\hline $\begin{array}{l}\text { Samal, } \\
\text { et al. (7) }\end{array}$ & 2015 & India & $\begin{array}{l}\text { Serie de } \\
\text { casos }\end{array}$ & 3 & 1 & $\begin{array}{c}8 \\
\text { semanas }\end{array}$ & $\begin{array}{l}\text { Dilatación y cure- } \\
\text { taje más tapona- } \\
\text { miento con balón } \\
\text { seguido de MTX } \\
50 \text { mg IM }\end{array}$ & 2 meses & Exitoso \\
\hline \multicolumn{10}{|c|}{ Histeroscopia } \\
\hline$\frac{\grave{3}}{2}$ & 毞 & है & 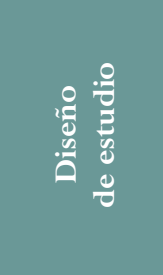 & 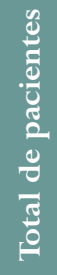 & 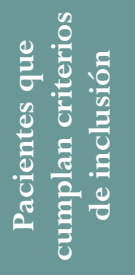 & 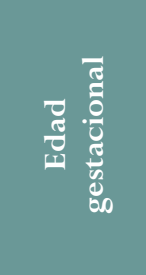 & 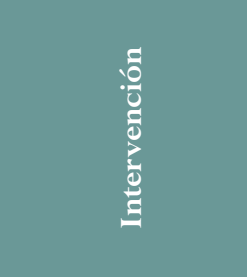 & 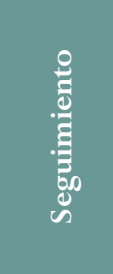 & 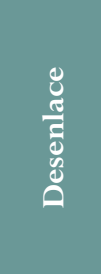 \\
\hline $\begin{array}{l}\text { Mangino, } \\
\text { et al. (16) }\end{array}$ & 2014 & Italia & $\begin{array}{l}\text { Reporte } \\
\text { de caso }\end{array}$ & 1 & 1 & $\begin{array}{c}6 \\
\text { semanas }\end{array}$ & $\begin{array}{l}\text { MTX intraamnió- } \\
\text { tico, dos dosis de } \\
\text { MTX sistémicas y } \\
\text { resección histeros- } \\
\text { cópica de rescate } \\
\text { ante no respuesta } \\
\text { al MTX }\end{array}$ & 2 meses & Exitoso \\
\hline $\begin{array}{l}\text { Scutiero, } \\
\text { et al. (19) }\end{array}$ & 2013 & Italia & Prospectivo & 5 & 5 & $\begin{array}{l}6 \text { semanas } \\
9 \text { semanas } \\
6 \text { semanas } \\
7 \text { semanas } \\
8 \text { semanas }\end{array}$ & $\begin{array}{l}\text { Embolización } \\
\text { arterial uterina } \\
\text { bilateral y poste- } \\
\text { rior excisión de } \\
\text { ectópico cervical } \\
\text { mediante histeros- } \\
\text { copia }\end{array}$ & 15 días & Exitoso \\
\hline $\begin{array}{l}\text { Tanos, } \\
\text { et al. (26) }\end{array}$ & 2019 & $\begin{array}{l}\text { Chipre } \\
\text { - Egipto }\end{array}$ & $\begin{array}{c}\text { Serie de } \\
\text { casos }\end{array}$ & 4 & 4 & $\begin{array}{l}7 \text { semanas } \\
6 \text { semanas } \\
6 \text { semanas } \\
5 \text { semanas } \\
4 \text { días }\end{array}$ & Histeroscopia & 1 día & Exitoso \\
\hline
\end{tabular}




\begin{tabular}{|c|c|c|c|c|c|c|c|c|c|}
\hline 를 & 遂 & $\stackrel{\mathscr{E}}{\mathrm{E}}$ & 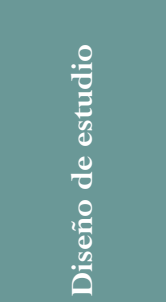 & 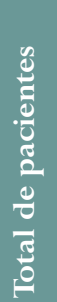 & 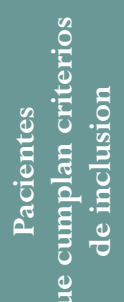 & 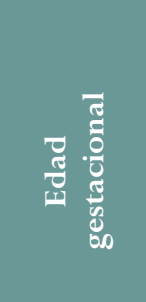 & $\begin{array}{l}\Xi \\
\frac{0}{0} \\
\frac{\pi}{0} \\
\frac{0}{0} \\
\Xi\end{array}$ & 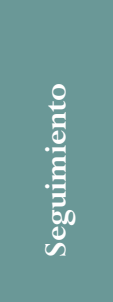 & 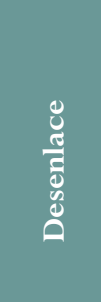 \\
\hline \multicolumn{10}{|c|}{ Histeroscopia } \\
\hline $\begin{array}{l}\text { Kim, } \\
\text { et al. }(27)\end{array}$ & 2008 & $\begin{array}{l}\text { Corea } \\
\text { del Sur }\end{array}$ & $\begin{array}{l}\text { Serie de } \\
\text { casos }\end{array}$ & 10 & 10 & $\begin{array}{c}<4 \\
\text { semanas } \\
<5 \\
\text { semanas } \\
6 \\
\text { semanas } \\
5 \text { semanas } \\
6 \text { días } \\
6 \text { semanas } \\
2 \text { días } \\
<5 \\
\text { semanas } \\
<5 \\
\text { semanas } \\
5 \text { semanas } \\
6 \text { días } \\
<4 \\
\text { semanas } \\
6 \text { semanas }\end{array}$ & Histeroscopia & 28 días & Exitoso \\
\hline \multicolumn{10}{|c|}{ Ligadura o embolización de arterias uterinas } \\
\hline$\frac{\grave{0}}{3}$ & 放 & है & 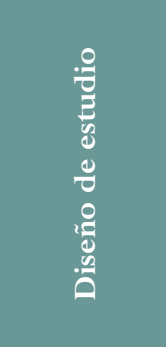 & 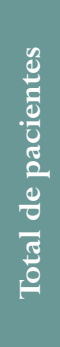 & 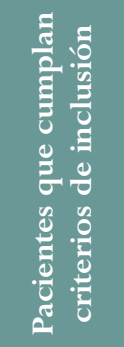 & 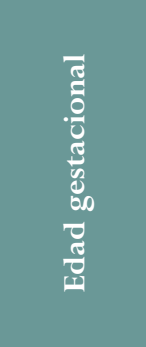 & $\begin{array}{l}\frac{\Xi}{0} \\
\frac{0}{0} \\
\frac{0}{0} \\
\text { ఏ }\end{array}$ & 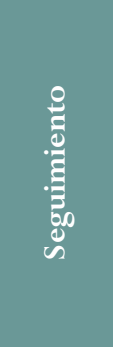 & 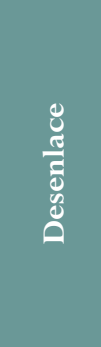 \\
\hline $\begin{array}{l}\text { Kung, } \\
\text { et al. (20) }\end{array}$ & 2004 & Taiwan & Prospectivo & 27 & 6 & $\begin{array}{c}9 \text { semanas } \\
9 \text { semanas } \\
6 \text { semanas } \\
6 \text { semanas } \\
6 \text { semanas } \\
6 \text { sema- } \\
\text { nas. }\end{array}$ & $\begin{array}{l}\text { Ligadura de } \\
\text { arterias uterinas } \\
\text { vía laparoscópica. }\end{array}$ & $\begin{array}{c}\text { 35-90 } \\
\text { días }\end{array}$ & Exitoso \\
\hline $\begin{array}{l}\mathrm{Hu}, \\
\text { et al. (24) }\end{array}$ & 2016 & China & Prospectivo & 19 & 19 & $\begin{array}{l}\text { Entre } 5.1 \\
\text { y } 11 \text { se- } \\
\text { manas de } \\
\text { gestación }\end{array}$ & $\begin{array}{l}\text { Embolización de } \\
\text { arterias uterinas } \\
\text { seguido de curetaje }\end{array}$ & $\begin{array}{c}59 \\
\text { meses } \\
\text { en } \\
\text { prome- } \\
\text { dio }\end{array}$ & Exitoso \\
\hline
\end{tabular}


deró exitoso en los diez casos, con un tiempo medio de remisión de la b-HCG de 8,8 \pm 3,6 semanas y un tiempo promedio de desaparición de la imagen ecográfica de masa cervical de 12,9 \pm 6,9 semanas. Dos pacientes presentaron complicaciones: una presentó ulceración oral severa e incremento de los títulos de b-HCG y requirió cambio de manejo a MTX local. Otra de las pacientes que recibió inyección local presentó hemorragia severa vaginal una semana después, que requirió transfusión de dos unidades de glóbulos rojos, curetaje y balón de sonda de Foley No. 18, inflado con $30 \mathrm{ml}$ endocervical con control de la hemorragia.

Martinez et al. (28) publicaron el reporte de caso de una paciente de 37 años con embarazo ectópico cervical temprano hemodinámicamente estable, se decidió realizar tratamiento con MTX con éxito, sin complicaciones.

De la loza Cava et al. (29) reportaron un caso de EC de 14 semanas, el cual recibió manejo inicialmente con MTX intramuscular (IM) en monodosis, con posterior deterioro hemodinámico y realización de histerectomía; en el posoperatorio cursó con evolución positiva, sin otras complicaciones clínicas. Se consideró un manejo conservador con MTX fallido.

Figueroa-Solano et al. (30) documentaron el caso de una paciente de 37 años con embarazo ístmicocervical, en quien se decidió la administración de MTX IV en multidosis por 4 semanas, con deterioro hemodinámico, que requirió histerectomía total abdominal sin complicaciones, por lo que se confirmó el manejo conservador fallido; la paciente egresó a los 39 días de hospitalización y la patología reportó un embarazo ístmico cervical y placenta increta.

Weibel et al. (31), en Canadá, publicaron dos casos clínicos de pacientes con EC de 5,6 y 7,4 semanas, quienes recibieron manejo conservador con MTX sistémico en multidosis intercalado con ácido folínico oral, con adecuada respuesta clínica, sin necesidad de más intervenciones, con lo que se ratificó el manejo conservador exitoso.
Mesogitis et al. (18) realizaron un estudio prospectivo de 9 pacientes con EC de entre 5,4 y 13,7 semanas, quienes recibieron manejo conservador con MTX intra-amniótico; 2 pacientes que presentaban embriocardia positiva en el seguimiento ultrasonográfico recibieron una segunda dosis de MTX intraamniótico. Tras dos ecografías con evidencia de feto muerto, se realizó curetaje cervical. En todas las pacientes se obtuvo tratamiento exitoso, sin necesidad de transfusión u otros procedimientos mayores.

Verma et al. (23), en un estudio retrospectivo de 25 pacientes con EC de 5 a 15 semanas, de las cuales 15 tenían feto vivo, un total de 19 recibieron MTX vía intramuscular. El tratamiento fue exitoso en todas ellas, pero cuatro tuvieron hemorragia aguda, una de ellas necesitó EAU con control de la hemorragia, otra paciente requirió tanto de manejo endovascular como de dilatación y curetaje y el uso de transfusión de hemoderivados, otra paciente presentó hemorragia crónica que requirió transfusión y otra presentó retención de partes fetales que requirió dilatación y curetaje. En ninguna fue necesaria la histerectomía. No se aclara si las pacientes que recibieron MTX fueron las que presentaron complicaciones.

Samal et al. (7) reportaron 3 casos de EC, uno de los cuales es correspondía a una mujer de 26 años quien presentaba embrión de 7 semanas con fetocardia, que recibió inyección de CLK y MTX. Se realizó seguimiento semanal con b-HCG encontrándose negativa a las 5 semanas; el tratamiento se consideró exitoso.

Murji et al. (15) publicaron un estudio retrospectivo de 27 pacientes de EC con edad gestacional media de 5 semanas. Todas recibieron MTX sistémico intramuscular ya sea en protocolo de monodosis o de multidosis. En 19 pacientes en quienes se encontró fetocardia positiva se utilizó KCL intraamniótico. Siete pacientes fueron sometidas a EAU, 2 de ellas como procedimiento preventivo de hemorragia, otras 2 para manejo de 
hemorragia severa y 3 como tratamiento combinado con dilatación y curetaje; en todas se consideró manejo exitoso.

Martínez et al. (32), en Cuba, documentaron el caso de una paciente con EC de aproximadamente 8 semanas de evolución, a la cual se le administró MTX en esquema de multidosis con persistencia del mismo en la ultrasonografía de seguimiento y con sangrado genital, por lo cual se decidió dar manejo definitivo con legrado del canal cervical sin complicaciones.

Tejero et al. (33) informan un caso en que se utilizó MTX vía intrasacular e intramuscular simultáneamente; al realizar el control ecográfico dos semanas después de la administración del fármaco, se observó la persistencia de un saco gestacional con embrión sin latido cardiaco a nivel cervical, por lo cual la paciente fue llevada a legrado obstétrico, sin complicaciones.

Taylor et al. (25), en su serie de 4 casos con EC, dieron tratamiento inicial con MTX IM con adecuada respuesta clínica en 3 pacientes, otra paciente fue sometida a EAU previa a la dilatación y curetaje, con adecuada evolución clínica final en los 4 casos, los cuales fueron manejos conservadores exitosos.

Samal et al. (35) informan el caso de una paciente de 29 años con EC de 6 semanas y 6 días, documentado por ultrasonido, con embrión vivo. Se inicia manejo con administración de $\mathrm{KCl}$ intrasacular amniótica y esquema de multidosis de MTX intramuscular $(1 \mathrm{mg} / \mathrm{kg})$ alternada con leucovorina $(0,1 \mathrm{mg} / \mathrm{kg})$. Al cuarto día la b-HCG era de 87.958 $\mathrm{mUI} / \mathrm{ml}$ y ecográficamente se evidenció una masa endocervical de 4,2 x 3,4x3,4 cm. Posterior a la cuarta dosis de MTX y leucovorina, la b-HCG fue de $42.955 \mathrm{mUI} / \mathrm{ml}$ y la imagen ecográfica endocervical no mostró cambios en tamaño. Se da de alta con controles semanales con b-HCG y ecografía transvaginal. A las dos semanas, la b-HCG era de 1267 $\mathrm{mUI} / \mathrm{ml}$ pero la imagen ecográfica mencionada persistía, por lo que debió reingresar para dilatación y curetaje el cual se realizó sin complicaciones; cua- tro semanas después del procedimiento la paciente presentó b-HCG negativa y ecografía transvaginal sin evidencia de colección endocervical. Se consideró manejo exitoso.

En total se identificaron 95 pacientes con EC tratados con MTX, la complicación más frecuente fue la hemorragia en 11 casos (12\%), en 25 se requirió tratamiento complementario (26\%), los más frecuentes fueron dilatación y legrado (8 casos) y EAU (10 casos), el cual se realizó de manera profiláctica en 7 casos, antes del legrado y del tratamiento con MTX. En 2 se requirió histerectomía abdominal, uno de los cuales fue un embarazo ístmico cervical.

\section{Tratamiento quirúrgico conservador}

Dilatación y curetaje endocervical: no se identificaron estudios en los que se realizara dilatación y curetaje (D\&C) como tratamiento único; hubo dos casos en los cuales se utilizó D\&C y taponamiento con sonda de Foley. De la Vega et al. (34) documentaron un caso de embarazo cervical de 7 semanas el cual recibió manejo conservador quirúrgico. La paciente, bajo anestesia general, fue infiltrada con $250 \mathrm{mi}-$ crogramos $(\mu \mathrm{g})$ de carboprost para una adecuada vasoconstricción, posteriormente se realizó cerclaje cervical y después se procedió a avanzar cureta al orificio cervical externo guiado por ecografía transabdominal; se obtuvo una gran cantidad de productos de la gestación cervical desde el interior del cérvix y tras dicho procedimiento, se colocó un balón de catéter de Foley de $30 \mathrm{ml}$ inflado con $25 \mathrm{ml}$ de solución salina en el canal cervical. No hubo complicaciones intra ni posoperatorias y el balón del catéter de Foley fue retirado a los 2 días sin complicaciones. Durante el seguimiento se confirmó manejo conservador quirúrgico exitoso.

Samal et al. (7) reportaron un caso tratado con $\mathrm{D} \& \mathrm{C}$, se trató de una paciente primigrávida de 27 años, con EC de 8 semanas, con imagen intracervical uterina de $4 \times 4 \mathrm{~cm}$, sugestiva de embarazo ectópico cervical, con una b-HCG en 1047 mUI/ $\mathrm{ml}$. Se realizó curetaje endocervical, seguido de 
colocación de balón de sonda de Foley No. 18, inflado con $40 \mathrm{ml}$ de solución salina, lográndose hemostasia, aunque requirió la administración de 3 unidades de glóbulos rojos y 1 una unidad de plasma fresco congelado. A las 24 horas se le retira el balón de Foley sin complicaciones, se le administra una dosis de MTX de $50 \mathrm{mg}$ IM y se le da egreso al tercer día. La patología informó vellosidades coriónicas, tejido decidual y glándulas más estroma cervical, lo que confirmó el diagnóstico. El seguimiento se realizó semanalmente con b-HCG con diagnóstico negativo a los 2 meses después del procedimiento, considerando tratamiento exitoso (tabla 2).

Histeroscopia: manguino et al. (16) reportaron un caso de manejo en una paciente de 40 años con antecedentes de enfermedad pélvica inflamatoria y concepción lograda por fertilización in vitro y transferencia embrionaria, quien presenta embarazo ectópico cervical con 6 semanas de amenorrea. La paciente recibió una dosis intrasacular amniótica guiada por ultrasonido de MTX y dosis sistémicas con mala respuesta y aumento de b-HCG de 1100 $\mathrm{mUI} / \mathrm{ml}$ a $4274 \mathrm{mUI} / \mathrm{ml}$, por lo cual se decidió resección histeroscópica sin ninguna otra intervención, con resultado exitoso y sin complicaciones.

Scutiero et al. (19) evaluaron 5 pacientes con EC entre 6 y 9 semanas de gestación, todas con actividad cardiaca embrionaria presente y rangos de b-HCG entre 15.482 y $74.684 \mathrm{mUI} / \mathrm{ml}$, combinando como tratamiento la embolización de arterias uterinas y posterior resección histeroscópica. Todas las pacientes tuvieron una estancia hospitalaria de 4 días y el resultado en todos los casos fue exitoso.

Tanos et al. (26) presentaron 4 pacientes con EC de 5 a 7 semanas de gestación y b-HCG entre 930 y $13.790 \mathrm{mUI} / \mathrm{ml}$. Todas fueron tratadas con histeroscopia como único manejo, usando como adyuvante vasoconstrictores (adrenalina diluida 1:40/2 cm y vasopresina diluida $1: 20 / 3,5 \mathrm{ml}$, infiltrada $5-7 \mathrm{~mm}$ en tres porciones adyacente al sitio de implantación del ectópico); el resultado en todas fue exitoso. El tiempo de estancia hospitalaria estuvo entre 8 y 24 horas, posterior al cual se les dio de alta a las pacientes sin complicaciones. Estos autores presentan una revisión de la literatura con 16 publicaciones de EC tratados con histeroscopia quirúrgica.

Kim et al. (27), en 10 pacientes hemodinámicamente estables, evaluaron la combinación de resección histeroscópica con infusión de $\mathrm{H}_{2} \mathrm{O}_{2}$ (peróxido de hidrógeno), e inyección intracervical de vasopresina $(0,2 \mathrm{U}-15 \mathrm{ml}$, aplicadas a las 3, 6, 9 y 12 posiciones del reloj); utilizaron el peróxido de hidrógeno bajo el concepto de que induce la muerte celular debido a la toxicidad del oxígeno. Las 10 pacientes incluidas se encontraban entre 4 y 6 semanas de gestación, con b-HCG entre 1561 y $47.629 \mathrm{mUI} / \mathrm{ml}$, ninguna recibió MTX. Todas las intervenciones fueron exitosas y a las 4 semanas de seguimiento todas las pacientes habían negativizado la b-HCG.

En total se identifican 20 pacientes manejadas con histeroscopia: en 14 se utilizó además manejo con vasopresina, en una de ellas posterior al uso de MTX y en EAU en 5 casos. En todos los casos la histeroscopia fue exitosa (tabla 2).

Embolización o ligadura de arterias uterinas: Kung et al. (20) describen 6 pacientes con EC, quienes fueron llevadas a manejo quirúrgico conservador con ligadura de arterias uterinas vía laparoscópica bajo anestesia general. Una vez los cirujanos identificaban la estructura vascular, se ligaba en su porción distal con material de sutura vicryl 1-0 hasta bloquear el flujo sanguíneo del útero y cérvix. Posterior al procedimiento laparoscópico, se realizó histeroscopia para localizar el saco gestacional a nivel cervical y resecarlo. En todos los casos el sangrado fue mínimo y ninguno tuvo complicaciones graves que requirieran histerectomía. El tiempo medio de recuperación de la menstruación en estas pacientes fue de 63 días.

Hu et al. (24), en un estudio retrospectivo de 19 pacientes con embarazo cervical, realizaron EAU seguida de curetaje a las 24-72 horas posteriores al procedimiento endovascular. Ninguna de las 19 
pacientes presentó sangrado vaginal u otras complicaciones asociadas en el posoperatorio. El 100\% de los casos tuvieron preservación uterina.

\section{CONCLUSIONES}

El tratamiento con MTX sigue siendo el tratamiento conservador más frecuentemente descrito en la literatura internacional. La dilatación y el curetaje con taponamiento endocervical puede ser una opción en el manejo de urgencia del EC en instituciones de atención primaria. En instituciones donde se dispone de tecnologías de alta complejidad, la embolización de arterias uterinas previa a los procedimientos quirúrgicos y la histeroscopia son opciones que se deben considerar. Dado que actualmente es posible el diagnóstico temprano del EC, se requieren estudios multicéntricos que comparen las diferentes alternativas de manejo para una adecuada evaluación de su seguridad y efectividad.

\section{REFERENCIAS}

1. Alkatout I, Honemeyer U, Strauss A, et al. Clinical diagnosis and treatment of ectopic pregnancy. Obstet Gynecol Surv. 2013;68:571-81. https://doi. org/10.1097 OGX.0b013e31829cdbeb

2. Schneider P, Drezin DH. Cervical pregnancy. Am J Obstet \& Gynecol. 1957;93: 27-40. https://doi. org/10.1016/0002-9610(57)90735-3

3. Hung TH, Jeng CJ, Yang YC, Wang KG, Lan CC. Treatment of cervical pregnancy with methotrexate. Int J Gynaecol Obstet. 1996;53(3):243-7. https://doi. org/10.1016/0020-7292(96)02653-7

4. Ushakov FB, Elchalal U, Aceman PJ, Schenker JG. Cervical pregnancy: Past and future. Obstet Gynecol Surv. 1997;52:45-59. https://doi.org/10.1097/00006254199701000-00023

5. Vela G, Tulandi T. Cervical pregnancy: The importance of early diagnosis and treatment. J Minim Invasive Gynecol. 2007;14:481-4. https://doi.org/10.1016/j. jmig.2006.11.012

6. Cunningham FG, Grant NF, Leveno KJ, Gilstrap LC III, Haut JC, Wenstrom KD, editors. Williams Obstetrics. 21st ed. McGraw Hill: New York; 1997. pp. 883-910.
7. Samal SK, Rathod S. Cervical ectopic pregnancy. J Nat Sci Biol Med. 2015;6(1):257-60. https://doi.org/10.4103/0976-9668.149221

8. Rock JA, Damario MA. Ectopic pregnancy. In: Rock JA, Jones HW III, editors. The Linde's operative gynaecology. 9th ed. USA: Lippincott Williams \& Wilkins; 2003. pp. 507-536.

9. Hosni MM, Herath RP, Mumtaz R. Diagnostic and therapeutic dilemmas of cervical ectopic pregnancy. Obstet Gynecol Surv. 2014;69(5):261-76. https://doi. org/10.1097/OGX.0000000000000062

10. Takashima M, Yamasaki M, Fujita I, et al. Enhanced magnetic resonance imaging in monitoring of conservative treatment of cervical pregnancy. J Obstet Gynaecol. 1995;21(6):545-50. https://doi.org/10.1111/j.1447-0756.1995.tb 00910.x

11. Shinagawa $S$, Nagayama $M$. Cervical pregnancy as a possible sequela of induced abortion. Report of 19 cases. Am J Obstet Gynecol. 1969;105:282-4. https:// doi.org/10.1016/0002-9378(69)90075-1

12. Oyer R, Tarakjian D, Lev-Toaff A, et al. Treatment of cervical pregnancy with methotrexate. Obstet Gynecol. 1988;71:469-71.

13. Ash S, Farrell SA. Hysteroscopic resection of a cervical ectopic pregnancy. Fertil Steril. 1996;66(5):842-4. https://doi.org/10.1016/S0015-0282(16)58649-X

14. Murji A, Garbodian K, Thomas J, Cruickshank B. Conservative management of cervical ectopic pregnancy. J Obstet Gynaecol Can. 2015;37:1016-20. https://doi. org/10.1016/S1701-2163(16)30051-2

15. Kung FT, Chang SY. Efficacy of methotrexate treatment in viable and nonviable cervical pregnancies. Am J Obstet Gynecol. 1999;181:1438-44. https://doi.org/10.1016/S0002-9378(99)70389-3

16. Mangino FP, Romano F, Di Lorenzo G, Buomono F, De santo D, Scrimin F, et al. Total hysteroscopic treatment of cervical pregnancy: The 2-step technique. J Minim Invasive Gynecol. 2019;26(6):1011-12. https://doi.org/10.1016/j.jmig.2019.01.009

17. Hung TH, Shau WY, Hsieh TT, Hsu JJ, Soong YK, Jeng CJ. Prognostic factors for an unsatisfactory primary methotrexate treatment of cervical pregnancy: A quantitative review. Hum Reprod. 1998;13(9):2636-42. https://doi.org/10.1093/humrep/13.9.2636 
18. Mesogitis S, Pilalis A, Daskalakis G, et al. Management of early viable cervical pregnancy. BJOG. 2005;112:409-11. https://doi.org/10.1111/j.14710528.2004.00447.x

19. Scutiero G, Nappi L, Matteo M, Balzano S, Macarini L, Greco P. Cervical pregnancy treated by uterine artery embolization combined with office hysteroscopy. Eur J Obstet Gynecol Reprod Biol. 2013; 166(1):104-6. https://doi.org/10.1016/j.ejogrb.2012.10.013

20. Kung FT, Lin H, Hsu TY, et al. Differential diagnosis of suspected cervical pregnancy and conservative treatment with the combination of laparoscopy-assisted uterine artery ligation and hysteroscopic endocervical resection. Fertil Steril. 2004;81:1642-9. https://doi. org/10.1016/j.fertnstert.2003.11.034

21. Kirk E, Condous G, Haider Z, et al. The conservative management of cervical ectopic pregnancies. Ultrasound Obstet Gynecol. 2006;27:430-7. https://doi. org/10.1002/uog.2693

22. Uludag SZ, Kutuk MS, Aygen EM, Sahin Y. Conservative management of cervical ectopic pregnancy: Single-center experience. J Obstet Gynaecol Res. 2017;42:1-6. https://doi.org/10.1111/jog.13362

23. Verma U, Goharkhay N. Conservative management of cervical ectopic pregnancy. Fertil Steril. 2009;91:671-4. https://doi.org/10.1016/j. fertnstert.2007.12.054

24. Hu J, Tao X, Yin L, Shi Y. Successful conservative treatment of cervical pregnancy with uterine artery embolization followed by curettage: a report of 19 cases. BJOG. 2016; 123 Suppl 3:97-102. https://doi.org/10.1111/1471-0528.14005

25. Taylor JE, Yalcinkaya TM, Akar ME. Successful conservative management of cervical ectopic pregnancy: a case series. Arch Gynecol Obstet. 2011;283(6):1215-7. https://doi.org/10.1007/s00404-010-1529-7

26. Tanos V, ElAkhras S, Kaya B. Hysteroscopic management of cervical pregnancy: Case series and review of the literature. J Gynecol Obstet Hum Reprod. 2019;48(4):247-53. https://doi.org/10.1016/j.jogoh.2018.05.001
27. Kim JS, Nam KH, Kim TH, Lee HH, Lee KH. Hysteroscopic management of cervical pregnancy with intrauterine irrigation with $\mathrm{H} 2 \mathrm{O} 2$. J Minim Invasive Gynecol. 2008;15:627-30. https://doi.org/10.1016/j. jmig.2008.06.006

28. Martínez RA, Quintero L, García CA, Fernández de Castro A. Embarazo ectópico cervical: diagnóstico preciso y enfoque de manejo médico. Reporte de caso. Univ Med. 2018;59(1):75-86. https://doi. org/10.11144/Javeriana.umed59-1.ecto

29. De la Loza Cava, LR, Arévalo JAM. Embarazo ectópico cervical. Cuando el tratamiento conservador falla. Reporte de un caso y revisión de la bibliografía. Ginecol Obstet Mex. 2012;80(10):668-72.

30. Figueroa J, Castillo HF, Gonzales SD, Vivas MR, Hurtado SM, et al. Embarazo ectópico istmico-cervical complicado con placenta increta: reporte de caso. Avan Biomed. 2012;1(2):82-6.

31. Weibel HS, Alserri A, Reinhold C, Tulandi T. Multidose methotrexate treatment of cervical pregnancy. J Obstet Gynaecol Can. 2012;34:359-62. https://doi. org/10.1016/S1701-2163(16)35217-3

32. Martinez Camilo RV, Perez Martínez MP, Torriente Hernandez B. Embarazo ectópico cervical tratado con metotrexate. Reporte de un caso. Rev Cubana Obstet Ginecol. 2000;26:87-90.

33. Tejero EL, Ortega S, Royo B, Andrés P, Lapresta M, Campillos JM. Gestación ectópica cervical tratada mediante punción ecoguiada intrasacular de metrotexato. Rev Chil Obstet Ginecol. 2010;75(5):325-8. https://doi.org/10.4067/S071775262010000 500009

34. De La Vega GA, Avery C, Nemiroff R, Marchiano D. Treatment of early cervical pregnancy with cerclage, carboprost, curettage, and balloon tamponade. Obstet Gynecol. 2007;109(2 Pt2):505-7. https://doi. org/10.1097/01.AOG.0000220599.74326.94

35. Samal S, Ghose S, Pallavee P, Porkkodi P. Successful management of live cervical ectopic pregnancy: A case report. J Clin Diagn Res. 2015;9(12):QD03-QD04). https://doi.org/10.7860/JCDR/2015/12230.6871_ 


\section{CONTRIBUCIÓN DE LOS AUTORES}

Henry Hernán-Bolaños: participó en la planeación del artículo y en la revisión de contenido del manuscrito final. Andrés Ricaurte-Sossa: participó en la junta médica donde se tomó la decisión de iniciar manejo conservador para la paciente y en la obtención de los datos de los estudios seleccionados.

Fabio Zarama-Márquez: participó en la junta médica donde se tomó la decisión de iniciar manejo conservador para la paciente, en el análisis y la selección de la literatura, y en la redacción del manuscrito.
Ruth Fajardo-Rivera: participó en la atención de la paciente y en la revisión de datos e información; participó en la redacción del manuscrito.

Ruben Chicaiza: participó en la planeación del artículo y en la estructuración de las partes del mismo, verificación de la calidad de la información y documentación de las referencias.

Carlos Andrés Guerrero-Mejía: participó en la búsqueda de imágenes diagnósticas, los datos de laboratorio y la descripción de las imágenes. Participó en la revisión del manuscrito. Andrés Ricaurte-Fajardo: participó en la recolección de literatura y en la revisión del manuscrito.

\section{Conflicto de intereses: ninguno declarado.}

